\title{
The Design of ERP Intelligent Sales Management System
}

\author{
Jianghui Liu ${ }^{\mathrm{a}, 1}$, Qiuyi Chen ${ }^{\mathrm{b}}$ and Yuexing Qiu ${ }^{\mathrm{c}}$ \\ ${ }^{a}$ Network and Information Center, Experimental Teaching Center, Guangdong \\ University of Foreign Studies, China \\ ${ }^{\mathrm{b}}$ School of Business, Guangdong University of Foreign Studies, China \\ ${ }^{\mathrm{c}}$ School of Economics and Trade, Guangdong University of Foreign Studies, China
}

\begin{abstract}
With the popularization of informatization, most companies have their own information management systems. However, faced with massive amounts of data, most companies cannot integrate and utilize its potential value. When traditional companies make sales forecasts, they usually purchase data completion, connect multiple data source channels, and then judge the future sales situation perceptually. This lack of data accumulation and analysis, and it is impossible to display the intrinsic value of the massive data in the system. Some companies will also build professional data analysis teams or seek help from third-party companies. But this will cause high expenses and greatly reduce sales expenses. This study combines artificial intelligence technology with ERP sales management system. We apply artificial intelligence, machine learning, cloud computing to the design and construction of intelligent ERP sales management system. It solves the core problems of enterprise product sales through the deep learning function of the sales system. It may predict enterprise sales and rationally allocate enterprise resources, increase product sales effectively, and help enterprises build effective modern management systems.
\end{abstract}

Keywords. ERP, Sales Management, Artificial Intelligence, Deep Learning

\section{$1 \quad$ Preface}

Enterprises should effectively use the huge amount of accumulated data. Enterprises may explore the value behind the data. This study will help enterprises improve the traditional ERP system. The study integrates big data, cloud computing, artificial intelligence into the traditional ERP system. And the new system may make sales forecasts for the enterprise. It will reduce inventory costs and improve business efficiency.

How do companies keep up with the development of technologies to improve the sales management? This study may give enterprises some enlightenment.

1 Corresponding Author: Jianghui Liu, Network and Information Center, Experimental Teaching Center, Guangdong University of Foreign Studies, China; Email: maggyliu1978@163.com 


\section{Studies review}

\subsection{Background}

In China, small and medium-sized enterprises have small production scales, weak technological research, development capabilities and weak anti-risk capabilities. The impact of the corona virus alone has caused many small and medium enterprises to close down. For such small and medium-sized enterprises, how to adapt to the ever-changing customer needs under the inherent background and how to keep up with the speed of economic globalization are all issues that worth considering. With the trend of economic globalization, the advantage of China's cheap labor is not existing. A large number of enterprises withdraw from my China, which has brought a rapid withdrawal of capital and technology. In response to its impact, China has begun to improve the competitiveness of enterprises through technological innovations and lower operating costs.

At present, managers only make some decisions based on former experience and decision makers' intuition, rather than based on information-rich data in the database. Meanwhile, it is difficult for traditional forecasting methods to take into account the information brought by complex and diverse data. Therefore, traditional forecasting methods have no way to meet the increasing requirements of enterprises for sales demand. The accuracy of traditional sales forecasting of enterprises is becoming lower and lower.

ERP intelligent sales forecast is only in its infancy in China, especially for small and medium-sized enterprises that have not yet had the concept of intelligent ERP. At present, companies have an urgent need for intelligent ERP to achieve accurate sales forecasts and enhance their competitiveness. The ERP intelligent sales system helps companies find reliable forecasting information from a large amount of historical data, analyze the correlation between products and customers, and analyze the correlation between products and suppliers, and then use machine learning to train the model to improve accuracy.

\subsection{Development status and shortcomings of ERP sales system at home and abroad}

The development of artificial intelligence is rapid in recent years. The demand of enterprises in the application of artificial intelligence and cloud platforms is becoming more and more popular. PwC's $22^{\text {nd }}$ Annual Global CEO Survey shows that $85 \%$ of CEOs believe that artificial intelligence will have a significant impact on companies and their business models in the next five years. However, the scope of application of artificial intelligence in enterprise management is still limited so far. Less than one-tenth of the CEO said that artificial intelligence is being widely used. In addition, according to a report released by Panorama Consulting in early 2020 , only $33 \%$ of suppliers use cloud services or provide cloud-based services. About $67 \%$ of companies still use local ERP systems. It shows that companies should use cloud-based ERP and it can take the lead in artificial intelligence. [1]

Both artificial intelligence and ERP can provide good services to enterprises. It can optimize and improve management decision-making. It can also improve the sales efficiency of enterprises. Therefore, the combination of the two will have broad development prospects. Cloud service providers have applied and promoted big data technologies, such as block chain and cloud computing at the same time. It uses 
machine learning to process massive amounts of data information. It has increased the efficiency of cloud sharing and the level of user demand for cloud sharing services. [2]

The previous research and project applications were mainly in the fields of financial management, salary management, warehouse management and so on. The previous research has less involved in sales management. With the continuous improvement of the market mechanism, the amount of relevant data information continues to grow rapidly. The importance of sales information management in the enterprise continues to increase. It is becoming one of the core tasks of the enterprises [3]. However, the sales management system still has some problems of single development means, such as its limited system service items and low technical content. It makes the overall application effect of the system and the actual business needs a large gap.

This study discusses the design of the ERP sales management system based on artificial intelligence technology. It uses the latest artificial intelligence, big data processing and analysis technology, cloud computing, blockchain to build an artificial intelligence-based sales management system in the ERP environment. It also solves the core problems of enterprise product sales. And it helps enterprises build an effective way of modern management system and realize the leapfrog development from relying on experience to make sales forecasts to technology.

\section{3}

\section{Theory and related technology}

\subsection{Artificial Intelligence}

Artificial intelligence completes identification, understanding, analysis, and decision-making through replacing humans with the development of human-related theories, technologies, and application systems. The popularity of mobile Internet, the rise of supercomputing and big data has brought the research of artificial intelligence into a new stage. The related applications have gradually penetrated into various fields of social life that play a role in assisting human work. [4]

The core of artificial intelligence research is machine learning. Computer derives laws can predict unknown new data by studying existing data. The computer has the ability to learn as intelligently as a person. Among them, machine learning is mainly divided into two categories: supervised learning and unsupervised learning. Supervised learning is mainly aimed at classification and regression problems. It finds rules in the data information given corresponding feature labels. Unsupervised learning is mainly oriented to cluster analysis and data dimensionality reduction [5]. It requires the computer to actively determine the characteristics of the data by looking for the rules in the data.

Artificial intelligence is widely used in sales. It has applications in personalized content, product recommendations, conversational artificial intelligence, predictive analysis and insights, and social listening and sentiment analysis. The application of artificial intelligence can only predict the total sales amount at present. It cannot predict the consumption quota of a single user. Also, there are problems that the massive data is difficult to manage and the data is redundant. It is difficult for enterprises to obtain intuitive results from the massive data. This project will focus on researching issues such as consumption forecasting [6]. This project can also realize functions such as 
workflow automation and consumption forecasting. It aims to help companies improve sales performance.

\subsection{Demand analysis of commodity sales management system}

Cloud computing technology is to simplify computer equipment. Various application software and their computing capabilities simplify the complicated work by providing services on the Internet. The rapid and widespread use of network technology makes the application environment of commercial software systems become very dependent in all walks of life on the Internet. Under the protection of the Internet, people only need to use a computer or mobile device. And they can access the Internet to use the application system anytime and anywhere. This breaks the shortcomings of the local system application limitations. It is also significantly saving the time for users to use the system to process work. Improve work efficiency. [7]

The combined application of ERP sales system and cloud service can greatly save the cost of enterprise system construction. It can improve the efficiency of system use and make enterprise management more systematic and intelligent.

\section{Demand analysis of commodity sales management system}

\subsection{Business needs}

The interface of the sales information management system needs to implement the concept of intelligent and personalized design. So that users can complete the management of related merchandise sales information by performing simple operations. The intelligent sales management system should have the economic feasibility of making the system work for a long time. It should also have the feasibility of the merchants using the system for actual operation. [8]

In addition to the corresponding basic functions, the intelligent sales management system also needs to have the function of sales forecasting. Therefore, the background production resources can be reasonably allocated to effectively solve the sales problems such as the disadvantage of hot-selling commodities and the backlog of unpopular commodities, which can reduce production costs and maximize the profits of enterprises [9]. The demand for sales forecasting function is to conduct deep learning on the law of historical sales data and scientifically predict the future sales of different products. Then it should find potential customers and ensure product sales through pushing advertisements. Finally, it should formulate corresponding production plans to allocate enterprises reasonably resources to increase sales.

\subsection{System design goals}

The general goals of the commodity sales management system design is shown in Figure 1. 


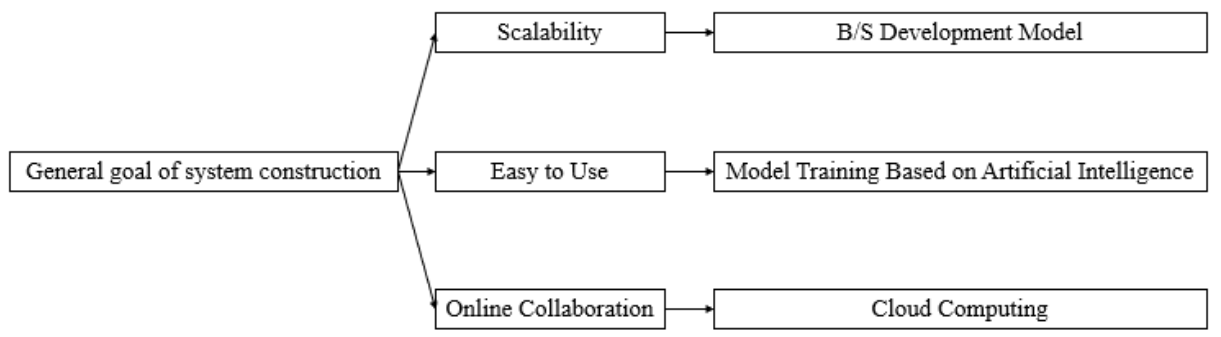

Figure 1. The overall goal of system construction

The system is optimized and rebuilt on the basis of the relevant technology of the original ERP system of the enterprise. Eventually, it will meet the needs of business and management. The goals of system construction are as follows: (1) To integrate the data of the original database of the enterprise, introduce data mining technology and make full use of the data accumulated by the enterprise to bring new profit growth points for the enterprise. (2) Using data mining technology and highly automated analysis of the original data of the enterprise to make inductive reasoning, mining potential patterns, predicting customer behavior, helping enterprise decision makers adjust market strategies and reducing risks. (3) Adopting the $\mathrm{B} / \mathrm{S}$ internal and external network combination mode to enable the system to realize the function of one-key prediction based on various dimensions.

\section{Design of commodity sales management system based on artificial intelligence}

Based on the above analysis of the sales system, this study divides the intelligent sales management system into multiple core modules for design. And it sorts out the core functions of each link in the specific division process, as is shown in Figure 2.

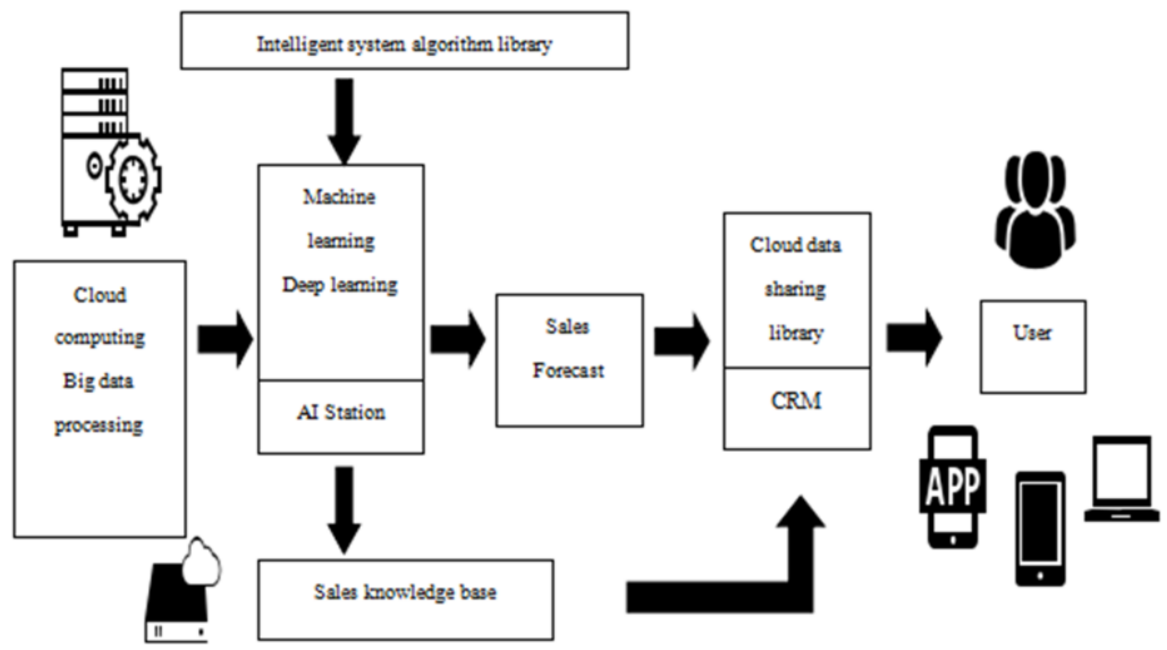

Figure 2 ERP intelligent sales system architecture 
- To support the application of large-scale artificial intelligence, we realize the application of data processing, model analysis, application reasoning and other functions through the operation of machine learning and deep learning. For data, we will first perform the following three steps: data preparation, mining operations, result expression and interpretation. In the data preparation stage, we call the data in the database and perform integrated operations on the data, which is to merge the data in the multi-file or multi-database operating environment, explain the semantic ambiguity, deal with the future in the data, and clear the dirty data. Then select the data, narrow the processing range, and improve the quality. After the data preprocessing operation, the data mining is in the procedure. In this process, we first decide how to generate hypotheses, then select the appropriate tools to explore the data, and finally verify the knowledge found. We use the model and continuously train the model to form in the mode of continuous optimization. The final process is to visualize and explain the results.

- We build a rich and diverse deep learning system architecture and machine learning algorithm library environment, such as Torch, CNTK, Cafe-MPI and TensorFlow. It can provide users with a full range of services.

- Through the implementation of cloud service resource management platform, deep learning management AI Station platform and application analysis tool Teye, the implementation of enterprise data information is effectively summarized, changed, adjusted, arranged and analyzed.

- Distribute differentiated computing resources based on the computing characteristics of machine learning, deep learning models and the related resource requirements are required. Taking the CPU cluster required for data preprocessing as an example, in order to complete the rapid preprocessing of model data, Nvidia P40 high-density GPU cluster can be used to complete rapid training for large-scale big data models for deep learning with inference recognition function. Moreover, we provide FPGA cluster to meet the requirements of high throughput and low latency processing. The cloud platform system as a whole provides high-speed cloud services with large capacity, high bandwidth, and low resource storage delay.

- Forecast product sales is based on machine learning. Firstly, we explore and confirm the distribution of product data and missing information through data analysis. Secondly, we use feature engineering to split columns containing complex data information into time nodes of day, week, month, and year, and clearly sell products through visualization technology. The amount of the forecast has the greatest impact on the characteristics. Through the algorithm to predict the sales situation of the seller in the next six months, the key data features are more intuitively displayed in a graphical arrangement. The performance of different single models in predicting customer consumption is compared horizontally. Finally, the models are combined linearly. And the linear combination model with the highest accuracy is selected from them.

- Predict product sales is based on BP network prediction algorithm. Neural network is a non-linear adaptive system that can self-find the internal connection of sales data through machine learning to achieve prediction. 
The algorithm is as follows:

The output of the i-th neuron in the hidden layer is:

$$
\mathrm{al}_{i}=f_{1}\left(\sum_{j=1}^{r} w l_{i j} p_{j}+b l_{i}\right)
$$

The output of the kth neuron in the output layer is:

$$
\mathrm{ak}_{2}=f_{2}\left(\sum_{i=1}^{s 1} w 2_{k i} a l_{i}+b 2_{k}\right)
$$

The defined error function is:

$$
\mathrm{e}=\frac{1}{2} \sum_{k=1}^{s 2}\left(t_{k}-a 2_{k}\right)^{2}
$$

Since the algorithm has an error between the actual output and the ideal in actual operation, the algorithm converges. Therefore, the algorithm is optimized, and the correlation analysis can be used to analyze the factors that most affect sales forecasts. The data of these factors and historical real data are analyzed again. If the correlation is high, the original sales model is proved to be feasible. Then the correlation coefficient will be further optimized until the predicted data is consistent with the real data (here the agreement refers to more than $95 \%$ ).

Performing correlation analysis on the algorithm and then adjusting and optimizing:

The adjustment formulas for the weight and threshold of the output layer can be expressed as:

$$
\begin{aligned}
& \mathrm{w} 2_{k i}(\mathrm{t}+1)=\mathrm{w} 2_{k j}(\mathrm{t})+\sigma \sum_{m=1}^{Q} \lambda 2_{k m} a l_{i m} \\
& \mathrm{~b} 2_{k i}(\mathrm{t}+1)=\mathrm{b} 2_{k}(\mathrm{t})+\sigma \sum_{m=1}^{Q} \lambda 2_{k m}
\end{aligned}
$$

The adjustment formulas of the hidden layer weight and threshold are respectively expressed as:

$$
\begin{aligned}
& \mathrm{wl}_{i j}(\mathrm{t}+1)=\mathrm{w} l_{i j}(\mathrm{t})+\sigma \sum_{m=1}^{Q} \lambda 1_{i m} p_{j m} \\
& \mathrm{bl}_{i j}(\mathrm{t}+1)=\mathrm{b} l_{i j}(\mathrm{t})+\sigma \sum_{m=1}^{Q} \lambda 1_{i m}
\end{aligned}
$$

Where $\mathrm{P}$ is the input sample; $\mathrm{Q}$ is the number of input samples; $t_{k}$ is the expected output of the network; $\sigma$ is the learning step size, $0<\sigma<1, \lambda 1$ is the hidden layer error transmission term; $\lambda 2$ is the output layer error transmission term.

Finally, enterprises can use cloud sharing as a data sharing library. Employees can obtain these materials through the mobile APP. The sharing tool provides a simple way to centrally manage file materials. It can authorize different user groups to view different materials. It frees people from the tedious work of making paper materials.

\section{Experimental results}

This experiment takes a company's product A as an example. It compares the sales of the product within six months when using the intelligent sales system and the traditional sales system. It verifies the improvement effect of the intelligent sales system on product sales. 
Firstly, we enter the sales data for the first six months of product A and make preparations for forecasting. The result is shown in Figure 3.

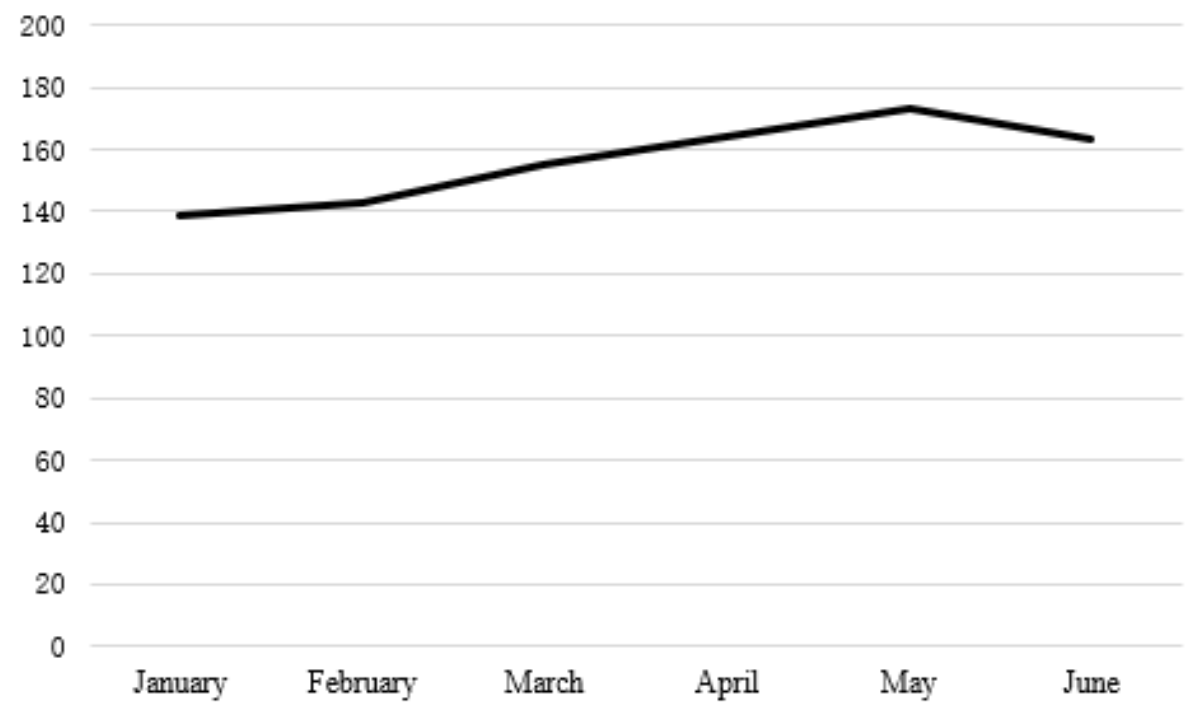

Figure 3 A company's sales of A products

From July to December, the intelligent sales system and the traditional sales system were used to observe the difference in product sales when applying two different systems. In the first six months of using the traditional sales system, the monthly sales volume of product A was between 139 and 173. The overall sales volume was low. In the second half of the year, in the control group, the traditional sales system was used. The result is shown in Figure 4.

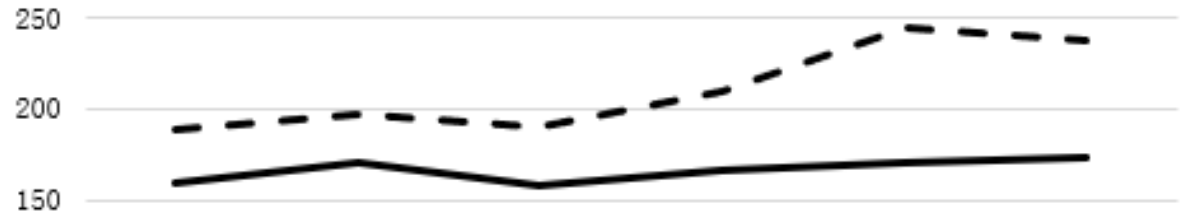

0

$$
\begin{gathered}
\text { July August } \quad \text { September } \\
\text { Traditional sales system }
\end{gathered}
$$

Figure 4 Comparison of sales of traditional and intelligent ERP sales systems 
The sales volume of the product remained the same as that of the previous June with an average of 166 products sold per month. After using the intelligent sales system, the sales volume of the modified products has been greatly increased. The use of the intelligent sales system improves customer satisfaction. Through sales forecasting, we understand customer needs and provide corresponding services in a timely manner. And we also reduce the loss of sales and increase customer loyalty. The use of intelligent sales system also allows companies to arrange production more effectively, and the improvement of the forecast level will directly determine the effectiveness of the production plan, and it can reduce inventory and safety stock. Sales forecasts would reduce inventory backlogs, and directly reduce safety stocks, improve inventory management. It would effectively increase sales to an average of 211 products sold per month, and improve corporate operating efficiency.

\section{Conclusion}

This study designs the ERP intelligent sales management system based on artificial intelligence technology. Through the analysis of the demand of the commodity sales system, it clarifies the sales forecasting functions that the intelligent sales management system should have, and then discusses the specific implementation technology and introduces the sales forecasting model. It determines the system architecture of the commodity sales information management platform. In the process of designing this system, the following conclusions are obtained: (1) Through the investigation of various enterprise sales department, we can fully understand the sales pain points. Then we summarize the actual needs of the enterprise and optimize the ERP sales system. Finally, we improve the efficiency of business operations. (2) When designing the architecture, the most important design part is to integrate artificial intelligence and cloud computing into the ERP sales management system, and build the system through BP algorithm, machine learning optimization model, cloud computing collaborative work and other methods. (3) Use .NET related technology to develop the company's sales information system and realize seamless connection of various departments, and the core modules of the system have reserved interface fields which are extensible. Experiments have proved that the intelligent ERP sales system effectively improves the efficiency of product sales management, simplifies sales communication links through deep learning, discovers potential customers, and effectively increases sales by pushing advertisements to them.

In the design process, although certain results have been achieved, but this study still has shortcomings. The intelligent ERP sales management system did not apply artificial intelligence to all aspects of the sales system.

\section{Acknowledgement}

This study was financially supported by the Undergraduate Innovation Training Project of Guangdong University of Foreign Studies in 2020. 


\section{References}

[1] Hicham A, Yassine T, Elyacoubi A, Lamia H. Forecasting Fuzzy Delphi and Hybrid intelligent system for ERP Architecture through the Scientific Private Cloud. International Journal of Computer Applications. 2018 April; 179(36):22-28.

[2] Mototake YI, Fukuda H, Ueda K. Creating an In-group Relation between Humans and Agents. Transactions of the Japanese Society for Artificial Intelligence. 2016 November; 31(6):AI30-J_1-10.

[3] Sahoo N, Dellarocas C, Srinivasan S. The Impact of Online Product Reviews on Product Returns. Information Systems Research. 2018 June; 29(3): DOI: 10.1287/isre.2017.0736.

[4] Deepak Alur, Dan Malks, John Crupi, Grady Booch, Martin Fowler. Core J2EE Patterns (Core Design Series): Best Practices and Design Strategies. Sun Microsystems, Inc. 2003.

[5] Cai, J. , Luo, J. , Wang, S. , \& Yang, S. . Feature selection in machine learning: a new perspective. NEUROCOMPUTING, 2018 July; 300:70-79.

[6] Avi Goldfarb, Daniel Trefler. Artificial Intelligence and International Trade. NBER Chapters, in: The Economics of Artificial Intelligence: An Agenda, National Bureau of Economic Research, Inc. 2018. 463-492.

[7] Christidis, K., Devetsikiotis, M.. Blockchains and smart contracts for the Internet of Things. IEEE Access 4. 2016 January; 2292-2303.

[8] Yoo M., Won Y. Study on Smart Automated Sales System with Blockchain-Based Data Storage and Management. In: Park J., Loia V., Yi G., Sung Y. (eds) Advances in Computer Science and Ubiquitous Computing. CUTE 2017, CSA 2017. Lecture Notes in Electrical Engineering, Singapore. Springer Press, 2017 December;. 474:734-740.

[9] Yu Q., Wang K., Strandhagen J.O., Wang Y. Application of Long Short-Term Memory Neural Network to Sales Forecasting in Retail-A Case Study. In: Wang K., Wang Y., Strandhagen J., Yu T. (eds) Advanced Manufacturing and Automation VII. IWAMA 2017. Lecture Notes in Electrical Engineering, Springer Press, 2018 February; Singapore. 451:11-17. 